\title{
Anatomical triangulation: from sparse landmarks to dense annotation of the skeleton in CT images
}

Marie Bieth ${ }^{12}$
marie.bieth@tum.de
Rene Donner

Bjoern Menze ${ }^{2}$

\author{
${ }^{1}$ Nuclear Medicin \\ Klinikum Rechts der Isar \\ Munich, Germany \\ ${ }^{2}$ Department of Computer Science \\ TU Munich \\ Munich, Germany \\ ${ }^{3}$ Department of Biomedical Imaging and Image-guided Therapy, \\ CIR Lab \\ Medical University of Vienna \\ Vienna, Austria
}

The automated annotation of bones that are visible in CT images of the skeleton is a challenging task which has, so far, been approached for only certain subregions of the skeleton, such as the spine. For example, in [4] and [2], methods are proposed for the annotation of the spine. In [5], a method is proposed to segment cartilage in the knee. In [6], a method is proposed to annotate the ribs. However, these methods are specific to a particular region of the skeleton and generalizing them to the whole skeleton, with a much larger field of view, a higher variability of the anatomy, and also variations in the positioning of the patient, remains an open task.

In this paper, we propose an approach for fully automatic dense annotation of substructures of the whole skeleton in CT images for staging bone tumour patients, where we want to localize anatomical substructures in order to estimate the local tumour load as visible from PET/CT images.

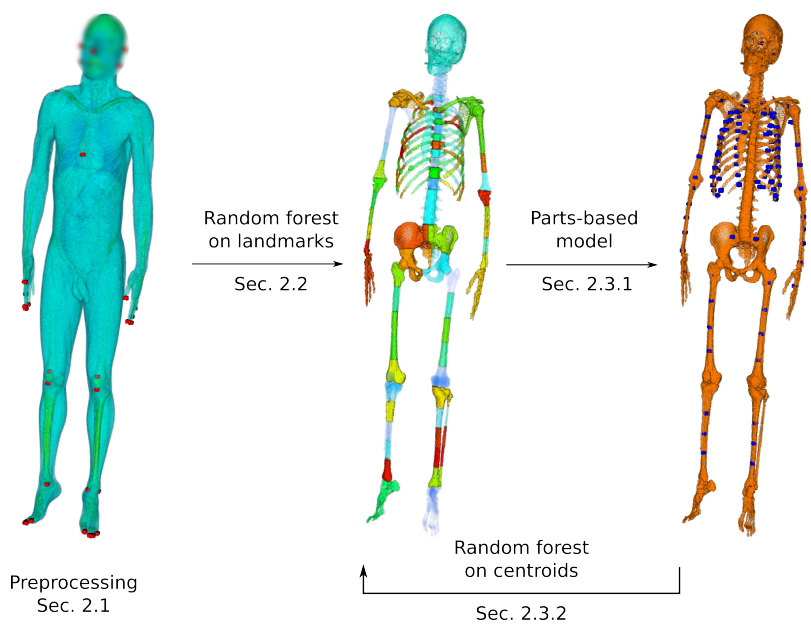

Figure 1: Description of the workflow. On the left, landmarks generated by [1] are shown. A first labelling (middle) is obtained using a Random Forest classifier. Centroids of each segment (right) are disambiguated by the parts-based model. They serve as new landmarks for another random forest classifier. The last two steps can be iterated.

As shown in Fig. 1, our method contains four main steps: the preprocessing, a first Random Forest classifier, a parts-based model and a second Random Forest classifier. The last two steps can be iterated.

Preprocessing: The bones are segmented from the CT image by adapting [3]. The intensities in the image are modelled as a mixture of two Gaussians and the resulting thresholding is regularized using graphcut. Landmarks distributed on the whole body are pre-filtered by a classifier and refined through a Hough regression model and a parts-based model of the global landmark topology as explained in [1].

Anatomical triangulation: A Random Forest classifier provides a first annotation of the skeleton. It uses as features: the triangulation features (signed distance to each landmarks in each direction), geodesic distances to selected landmarks, mean image intensity and bone proportion around the center voxel, position in the image.

Centroid regularization: The position of the centroid of each substructure of the skeleton is disambiguated by a Conditional Random Field
(CRF), whose unary and binary potentials use global anatomical knowledge. The best candidate centroid for each substructure is computed by minimizing the energy of the CRF.

Iterative triangulation: The centroids computed in the previous step are used as new, evenly distributed landmarks for a new Random Forest classifier based on distance features. The resulting probability distribution can either be converted to a hard labeling or used as input for a new centroid regularization step.

We have evaluated our method on 18 whole body CT scans. 136 bones or bone segments were annotated manually (example with shuffled labels in Fig. 1 middle). 57 landmarks were computed for each dataset (examples in Fig. 1 left). The test were run using six-fold cross-validation. Our method achieves a mean overall weighted Dice Score of 90.54.

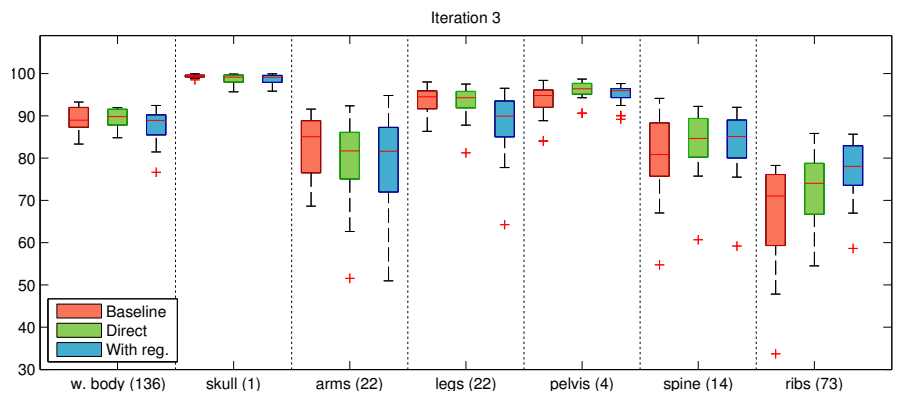

Figure 2: Weighted Dice scores for the segmentation of different groups of substructures by the baseline-random forest (red), after 3 iterations without centroid regularization (green), after three iterations with centroid regularization (blue). The total number of substructures of each group is given in brackets.

[1] René Donner et al. Global localization of 3D anatomical structures by pre-filtered Hough Forests and discrete optimization. Medical Image Analysis, 17(8):1304-1314, 2013.

[2] Ben Glocker et al. Vertebrae localization in pathological spine CT via dense classification from sparse annotations. In Proc. MICCAI 2013, pages 262-270. Springer, 2013.

[3] Yan Kang et al. A new accurate and precise 3-D segmentation method for skeletal structures in volumetric CT data. IEEE Transactions on Medical Imaging, 22(5):586-598, 2003.

[4] Stefan Schmidt et al. Spine detection and labeling using a parts-based graphical model. In Information Processing in Medical Imaging, pages 122-133. Springer, 2007.

[5] Quan Wang et al. Semantic context forests for learning-based knee cartilage segmentation in 3D MR images. In Medical Computer Vision. Large Data in Medical Imaging, pages 105-115. Springer, 2014.

[6] Dijia Wu et al. A learning based deformable template matching method for automatic rib centerline extraction and labeling in CT images. In Proc. CVPR 2012, pages 980-987. IEEE, 2012. 